\title{
Particle swarm optimization method in initialization of wavelet neural network model for fed-batch bioprocesses
}

\begin{abstract}
Wavelet neural network is an alternative to artificial neural network in empirical modeling of industrial processes due to efficient initialization of network parameters that reduces training time. In this paper, particle swarm optimization methods are used for initialization of dilation and translational parameters in two wavelet neural network models. Dissolved oxygen models are constructed from real bioprocess data of pilot scale fed-batch bioreactor in polyhydroxyalkanotes (PHA) production and an industrial-scale fed-batch bioreactor in penicillin production. Simulation output of dissolved oxygen and initial mean square error (IMSE) show that the distance and error between initialization and training data are small in PSO method compared to random and heuristic methods. This ensures training phase start very close to target data.
\end{abstract}

Keyword: Wavelet neural network; Particle swarm optimization; Fed-batch bioreactor 